\title{
Accuracy of the clinical diagnosis of dementia with Lewy bodies (DLB) among the Italian Dementia Centers: a study by the Italian DLB study group (DLB-SINdem)
}

\author{
Mirella Russo ${ }^{1}$ - Claudia Carrarini ${ }^{1} \cdot$ Angelo Di lorio $^{2} \cdot$ Raffaello Pellegrino $^{2}$ - Amalia Cecilia Bruni ${ }^{3}$. \\ Salvatore Caratozzolo ${ }^{4}$. Annalisa Chiari ${ }^{5}$. Stefano Pretta ${ }^{6}$. Camillo Marra ${ }^{7}$. Maria Sofia Cotelli ${ }^{8}$. Andrea Arighi ${ }^{9}$. \\ Giorgio G. Fumagalli ${ }^{9}$. Tatiana Cataruzza ${ }^{10}$. Francesca Caso ${ }^{11}$. Cristina Paci ${ }^{12}$. Mara Rosso ${ }^{13}$. Serena Amici ${ }^{14}$. \\ David Giannandrea ${ }^{15}$. Andrea Pilotto ${ }^{4}$ Simona Luzzi ${ }^{16}$. Annalisa Castellano ${ }^{17}$. Fabrizia D'antonio ${ }^{18}$. \\ Antonina Luca ${ }^{19}$. Giorgio Gelosa ${ }^{20}$ - Tommaso Piccoli $^{21} \cdot$ Marco Mauri $^{22} \cdot$ Federica Agosta $^{23}$. Claudio Babiloni ${ }^{24,25}$. \\ Barbara Borroni ${ }^{4} \cdot$ Marco Bozzali $^{26} \cdot$ Massimo Filippi $^{23} \cdot$ Daniela Galimberti ${ }^{9}{ }^{27} \cdot$ Roberto Monastero ${ }^{28}$. \\ Cristina Muscio ${ }^{29}$. Lucilla Parnetti ${ }^{30}$. Daniela Perani ${ }^{31}$. Laura Serra ${ }^{32}$. Vincenzo Silani ${ }^{33}$. Pietro Tiraboschi ${ }^{29}$. \\ Annachiara Cagnin ${ }^{34}$. Alessandro Padovani ${ }^{4} \cdot$ Laura Bonanni $^{2}$. on Behalf of the Italian Dementia with Lewy \\ bodies Study Group (DLB-SINdem)
}

Received: 9 December 2021 / Accepted: 25 February 2022 / Published online: 4 March 2022

(c) The Author(s) 2022, corrected publication 2022

\begin{abstract}
Introduction Dementia with Lewy bodies (DLB) may represent a diagnostic challenge, since its clinical picture overlaps with other dementia. Two toolkits have been developed to aid the clinician to diagnose DLB: the Lewy Body Composite Risk Score (LBCRS) and the Assessment Toolkit for DLB (AT-DLB). We aim to evaluate the reliability of these two questionnaires, and their ability to enhance the interpretation of the international consensus diagnostic criteria.

Methods LBCRS and AT-DLB were distributed to 135 Italian Neurological Centers for Cognitive Decline and Dementia (CDCDs), with the indication to administer them to all patients with dementia referred within the subsequent 3 months. We asked to subsequently apply consensus criteria for DLB diagnosis, to validate the diagnostic accuracy of the two toolkits. Results A total of 23 Centers joined the study; 1854 patients were enrolled. We found a prevalence of possible or probable DLB of $13 \%$ each (26\% total), according to the consensus criteria. LBCRS toolkit showed good reliability, with a Cronbach alpha of 0.77 , stable even after removing variables from the construct. AT-DLB toolkit Cronbach alpha was 0.52 and, after the subtraction of the "cognitive fluctuation" criterion, was only 0.31 . Accuracy, sensitivity, and specificity were higher for LBCRS vs. AT-DLB. However, when simultaneously considered in the logistic models, AT-DLB showed a better performance $(p<0.001)$. Overall, the concordance between LBCRS positive and AT-DLB possible/probable was of 78.02\% Conclusions In a clinical setting, the LBCRS and AT-DLB questionnaires have good accuracy for DLB diagnosis.
\end{abstract}

Keywords Dementia with Lewy bodies · Diagnostic toolkits $\cdot$ Consensus criteria $\cdot$ Clinical diagnosis $\cdot$ Dementia Cognitive impairment $\cdot$ Diagnostic accuracy

Mirella Russo and Claudia Carrarini contributed equally to this work.

Laura Bonanni

1.bonanni@unich.it

Extended author information available on the last page of the article

\section{Introduction}

Dementia with Lewy bodies (DLB), the second most common neurological cause of dementia after Alzheimer's disease $(\mathrm{AD})$, is characterized by cognitive fluctuations $(\mathrm{CF})$, visual hallucinations $(\mathrm{VH})$, parkinsonism, and REM sleep behavior disorder (RBD), which are considered as its core clinical features [1]. The accuracy of the clinical diagnosis of DLB is however not satisfactory, as the clinical presentation may overlap with $\mathrm{AD}$ [2]. 
The recent efforts of the researchers focusing on DLB have been addressed toward the identification of biomarkers which could help diagnose DLB as compared with AD.

Great emphasis has recently been placed on the necessity to identify more sensitive and specific diagnostic markers and to define the prodromal stage of DLB in order to put in place timely pharmacological and management interventions [3].

This research stream is witnessed by the recent flourishing of International Consortia on DLB (E-DLB, American Lewy Body Dementia (LBD) association, ISTAART LBD Professional Interest Area (PIA)). The efforts of the Consortia are aimed to overcome the challenges in recruiting sufficiently large and unbiased cohorts, and to identify which diagnostic instruments are sensitive to change in DLB.

Toward these aims, the Italian Neurological Society for dementia (SINdem) promoted the constitution of an Italian DLB study group. The general objectives of the study group were defined as follows:

a. To improve DLB identification by physicians working in Centers for Cognitive Decline and Dementia (CDCDs).

b. To identify the DLB cohorts available in Italy and develop an efficient method of data collection.

A first survey conceived to identify the DLB cohorts available in Italy was performed in 2016 [4]. The CDCDs were asked to answer a semi-structured questionnaire, which investigated the following: (1) incidence and prevalence of DLB; (2) clinical assessment; (3) relevance and availability of diagnostic tools; (4) pharmacological management of cognitive, motor, and behavioral disturbances; (5) causes of hospitalization, with specific focus on delirium and its treatment. Overall, 135 centers (23.6\% of all CDCDs) contributed to that first survey. A total of 5624 patients with DLB were followed at the time of the survey by the 135 centers in a year (2042 of them were new referrals). The percentage of DLB patients among neurodegenerative dementia was $27 \pm 8 \%$.

The prevalence of DLB in the Italian dementia population appeared therefore to be far higher than the one reported in literature, which ranges from 5 to $15 \%$ [1].

We explained that result by judging a semi-structured questionnaire as insufficiently accurate to avoid catching up cases of mixed dementia, which could be wrongly classified as DLB, in a country where neurodegenerative disease cases are not neuropathologically confirmed.

The aim of the present study is to re-evaluate the prevalence of DLB patients by using two standardized questionnaires, based on clinical diagnostic criteria for DLB. The questionnaires were specifically designed to address the problem of inadequate recognition and diagnosis of DLB $[5,6]$.
The first questionnaire, the Lewy Body Composite Risk Score (LBCRS) [7], was designed to improve the ability to detect DLB in clinical and research populations and to increase the likelihood of determining whether Lewy bodies are a contributing pathology to the dementia syndrome. The LBCRS was derived from clinical features in autopsyverified cases of healthy controls, AD, DLB, and Parkinson disease (PD) with and without dementia (PDD). The LBCRS was tested in comparison with gold standard measures of cognition, motor symptoms, function, and behavior.

The second questionnaire, the Assessment Toolkit for DLB (AT-DLB) [8], was developed to be aligned with the standard diagnostic criteria for DLB [1], and to be applied by clinicians in regular clinical services and easily integrated into routine care.

\section{Methods}

In this cross-sectional observational study, the two questionnaires were distributed in March 2019 to the 135 neurological CDCDs, which participated in the previous survey. The CDCDs included were evenly distributed over the country from north to south. Out of the 23 participating Centers, 10 CDCDs (43\%) are located within Neurology Units of University Hospitals, 5 CDCDs (22\%) belong to Scientific Institute for Research, Hospitalization and Healthcare (IRCSS), 5 CDCDs (22\%) are placed in Neurology Units of Non-Academic Hospitals, and 3 CDCDs (13\%) belong to territorial out-patient clinics.

We asked to administer the surveys to all the patients with dementia (Mini-Mental State Examination (MMSE) score $<24)$ [9] referred to the Centers in the subsequent 3 months, independently from the initial suspected diagnosis and from the final diagnoses.

All Centers were trained to apply the most recent diagnostic criteria for DLB [1].

More specifically, the neurologists of each Center were instructed to carry out in each patient a physical and neurological examinations.

The presence of cognitive fluctuations was confirmed by detailed semi-structured interview and quantified using the Clinician Assessment of Fluctuations questionnaire [10]. Visual hallucinations were determined by detailed interview with the patient and caregiver followed by confirmation and quantification according to the Neuropsychiatric Inventory [11]. Parkinsonism was diagnosed by the Motor part (part III) of the Unified Parkinson's Disease (PD) Rating Scale (UPDRS) [12]. Symptoms of RBD were assessed by interview and scored according to the Mayo Sleep Questionnaire [13]. The application of the DLB diagnostic criteria based on the aforementioned tests took about $60 \mathrm{~min}$. 
Each of the two questionnaires (LBCRS and AT-DLB) were administered to patients in random order.

The administration of the questionnaires took between 30 and 60 min, depending on the compliance of the patients and caregivers, and on the severity of the clinical picture. Considering the items included in each questionnaire, the aim was to compare the results of either LBCRS or ATDLB with the recent criteria. Where considered clinically appropriate, the patients underwent neuroimaging and neurophysiology assessments, as DaT-SPECT, myocardial scintigraphy, FDG-PET for detection of the Cingulate Island Sign, Quantitative EEG, polysomnography to confirm RBD. All procedures were in accordance with the ethical standards of the institutional and national research committee and with the Helsinki Declaration. Informed consent was obtained by all participants.

\section{Lewy Body Composite Risk Score}

The LBCRS [7] evaluates the presence of four motor and six non-motor symptoms within the last 6 months. Motor signs include slowness, rigidity, postural instability, and resting tremor. Non-motor symptoms are the following: excessive daytime sleepiness, illogical thoughts, frequent episodes of staring, visual hallucinations ( $\mathrm{VH}$ ), enacted dreaming, and autonomic dysfunctions.

The symptoms were considered as present if they occurred at least three times during the 6 months preceding the clinical investigation.

A global score equal or superior to 3 indicates a probable DLB diagnosis (LBCRS positive), whereas a score ranging from 0 to 2 is not suggestive of DLB diagnosis (LBCRS negative).

\section{Assessment Toolkit for Dementia with Lewy Bodies}

The Assessment Toolkit for DLB [8] is based on a series of specific questions carried out to identify core and suggestive features of DLB. Beyond the evidence of global cognitive decline, four domains are investigated (core clinical features): $\mathrm{CF}, \mathrm{VH}, \mathrm{RBD}$, and parkinsonism. The toolkit includes a questionnaire that is administered by the rater to the patient and the caregiver, and a short neurological exam to determine the 5-item Unified Parkinson's Disease Rating Scale (UPDRS) score. Moreover, the presence of dopaminergic deficit in basal ganglia on SPECT/PET, low uptake on metaiodobenzylguanidine (MIBG) myocardial scintigraphy, or polysomnography confirmation of RBD is evaluated. These features are considered as indicative biomarkers. A diagnosis of probable DLB is made if either two core features or one core and one indicative feature are identified. A diagnosis of possible DLB is considered if one feature is satisfied.

\section{Statistical analysis}

Statistical analysis was performed using Statistical Analysis Software (SAS).

Data were reported as mean \pm standard errors, for continuous variables, and as absolute number and percentage for categorical ones.

To assess the differences in the prevalence of symptoms in the three study groups, logistic models were used, where the absence of DLB study group was the reference. Moreover, to test whether the magnitude of association of each risk factor with DLB diagnosis differed between the 2 DLB group (probable and possible), the equivalence of the odds ratios (ORs) was computed by a Mantel-Haenszel $\chi^{2}$ statistic based on the weighted sum of the squared deviations of the stratum-specific log ORs from their weighted mean [14].

The statistical agreement of LBCRS and AT-DLB with the most recent diagnostic criteria of DLB was calculated. To consider how each variable reflects the reliability of a scale with standardized variables, the standardized alpha coefficient (Cronbach alpha) was also estimated. If the standardized alpha decreases after removing a variable from the model, that variable is strongly correlated with other variables included in the scale and contributes to the reliability of the scale. Conversely, if the standardized alpha increases after removing a variable from the model, removing that variable makes the scale more reliable [15]. The rate of concordance between the DLB clinical criteria [16] and the two toolkits was evaluated by accuracy, sensitivity, and specificity calculation. Logistic regression models were applied to calculate receiver operating characteristics (ROC) and to estimate the area under the curve (AUC). In the models, DLB criteria were the dependent variable while LBCRS and AT-DLB were the independent variables, which were simultaneously considered. When ROC was estimated for the "possible DLB" group, those classified as "probable DLB" were excluded from the analysis, and vice versa.

\section{Results}

Twenty-three CDCDs joined the research and performed the survey. Of the 2006 patients enrolled, 152 (7.58\%) subjects were excluded because of missing data. Among the 1854 remaining patients, 1048 (56.53\%) were female and the mean age of the sample was $75.06 \pm 14.58$ years. MMSE was $16.4 \pm 7.1$. All the patients underwent computed tomography/magnetic resonance imaging.

Seventy-four patients (4.7\%) underwent DaT-SPECT, 5 patients $(0.3 \%)$ underwent myocardial scintigraphy, 51 patients $(2.8 \%)$ underwent FDG-PET for detection of the Cingulate Island Sign, 29 patients (1.6\%) underwent 
quantitative EEG, and 5 patients $(0.3 \%)$ underwent polysomnography to confirm RBD.

Our results showed a prevalence of possible or probable DLB of $13 \%$ each (26\% total), according to the consensus criteria (Table 1).

\section{Lewy Body Composite Risk Score}

Applying the LBCRS in the study sample, 555 (30.66\%) patients were classified as DLB (LBCRS positive). The Cronbach alpha showed good reliability of the scale (0.77). Indeed, removing variables from the model, the alpha coefficient ranged from 0.74 to 0.76 .

Table 2 reports the frequencies of all the features included in the assessment, according to diagnosis reached using clinical DLB criteria. Among all items, those related to $\mathrm{VH}$ and RBD (questions 8 and 9) reported a higher ORs for diagnosis of probable DLB (VH-Q8: 40.61, 95\%CI: 27.48-60.01; RBD-Q9: 31.31, 95\%CI: 21.10-46.46).

Table 1 Patient diagnoses according to the consensus criteria for DLB (McKeith et al. 2017) at the two times of assessment

\begin{tabular}{lll}
\hline Patient diagnoses & $\begin{array}{l}\text { Consensus criteria } \\
\text { First assessment }\end{array}$ & $\begin{array}{l}\text { Consensus criteria } \\
\text { Second assessment }\end{array}$ \\
\hline No DLB & $73.46 \%$ & $73.03 \%$ \\
Possible DLB & $13.43 \%$ & $13.75 \%$ \\
Probable DLB & $13.11 \%$ & $13.21 \%$ \\
\hline
\end{tabular}

Abbreviations: $D L B$, dementia with Lewy bodies

\section{Assessment Toolkit for Dementia with Lewy Bodies}

After performing AT-DLB, 445 (24.59\%) patients were diagnosed as possible-DLB, whereas $322(17.79 \%)$ were probable DLB. For AT-DLB, the Cronbach alpha was of 0.52 . Only when removing the item " $\mathrm{CF}$," the alpha coefficient decreased to 0.31 .

Table 3 shows the frequencies of symptoms, considered in this scale, according to the recent DLB criteria. Among all items, those related to $\mathrm{CF}$ and $\mathrm{VH}$ (questions 1 and 7-10) were associated with the five highest ORs for diagnosis of probable DLB (CF-Q1: 52.73, 95\%CI: 36.06-77.09; RBD-Q7: 21.51, 95\%CI: 13.43-34.47; RBDQ8: 29.28, 95\%CI: 18.93-45.29; RBD-Q9: 42.79, 95\%CI: 28.13-64.18; RBD-Q10: 35.50, 95\%CI: 24.20-52.07).

\section{Comparison between Assessment Toolkit for Dementia with Lewy Bodies and Lewy Body Composite Risk Score}

The percentage of agreement between AT-DLB possible and LBCRS positive was $32.43 \%$, whereas the concordance between AT-DLB probable and LBCRS positive was higher (45.59\%). Overall, the concordance between LBCRS positive and AT-DLB possible/probable was of $78.02 \%$ (see Table 4 for the comparison between the two assessments). The agreement between LBCRS negative and AT-DLB negative was $88.30 \%$.

Table 5 shows the discrepancies between the two toolkits for attribution of patients to DLB diagnosis. Among all features, $\mathrm{CF}$ and $\mathrm{VH}$ showed a higher ORs for categorization of AT-DLB negative and LBCRS positive.

Table 2 Frequencies of all features included in the LBCRS in comparison with diagnostic criteria

\begin{tabular}{|c|c|c|c|c|c|c|}
\hline & No DLB & Possible DLB & $\begin{array}{l}\text { OR }(95 \% \mathrm{CI}) \\
\text { No vs. possible }\end{array}$ & Probable DLB & $\begin{array}{l}\text { OR }(95 \% \text { CI }) \\
\text { No vs. probable }\end{array}$ & $p$-value \\
\hline Patients $(n)$ & 1342 & 233 & & 235 & & \\
\hline Q1 Slowness & $258(19.2)$ & $99(42.5)$ & $3.1(2.3-4.2)$ & $174(74.0)$ & $12.0(8.7-16.5)$ & $<0.001$ \\
\hline Q2 Rigidity & $201(15.0)$ & $128(54.9)$ & $6.9(5.1-9.3)$ & $168(71.5)$ & $14.2(10.3-19.6)$ & $<0.001$ \\
\hline Q3 Postural instability & $190(14.2)$ & $74(31.8)$ & $2.8(2.1-3.9)$ & $110(46.8)$ & $5.3(4.0-7.2)$ & 0.003 \\
\hline Q4 Rest tremor & $57(4.3)$ & $29(12.5)$ & $3.2(2.0-5.1)$ & $67(28.5)$ & $9.0(6.1-13.3)$ & 0.004 \\
\hline Q5 Day sleepiness & $159(11.8)$ & $54(23.2)$ & $2.3(1.6-3.2)$ & $126(53.6)$ & $8.6(6.3-11.7)$ & $<0.001$ \\
\hline Q6 Illogical thinking & $287(21.4)$ & $72(30.9)$ & $1.6(1.2-2.3)$ & $122(51.9)$ & $4.0(3.0-5.3)$ & $<0.001$ \\
\hline $\begin{array}{l}\text { Q7 Episodes of absence or star- } \\
\text { ing off into space }\end{array}$ & $231(17.2)$ & $77(33.1)$ & $1.8(1.3-2.4)$ & $116(49.4)$ & $4.0(3.0-5.3)$ & $<0.001$ \\
\hline Q8 Visual hallucination & $47(3.5)$ & $54(23.2)$ & $8.3(5.5-12.7)$ & $140(59.6)$ & $40.6(27.5-60.0)$ & $<0.001$ \\
\hline Q9 Dreams enacted & $44(3.3)$ & $44(18.9)$ & $6.9(4.4-10.7)$ & $121(51.5)$ & $31.3(21.1-46.5)$ & $<0.001$ \\
\hline Q10 Orthostatic hypotension & $28(2.1)$ & $7(3.0)$ & $1.5(0.6-3.7)$ & $51(21.7)$ & $13.0(8.0-21.2)$ & $<0.001$ \\
\hline
\end{tabular}

Data are reported as number of patients, $n(\%) ; C I$, confidence interval; $O R$, odds ratio; $Q$, questions. The $p$-values* refer to test of comparison of the OR 
Table 3 Frequencies of all features included in AT-DLB in comparison with diagnostic criteria

\begin{tabular}{|c|c|c|c|c|c|c|}
\hline & No DLB & Possible DLB & $\begin{array}{l}\text { OR }(95 \% \mathrm{CI}) \\
\text { No DLB vs possible }\end{array}$ & Probable DLB & $\begin{array}{l}\text { OR }(95 \% \text { CI }) \\
\text { No DLB vs probable }\end{array}$ & $p$-value \\
\hline Patients $(n)$ & 1342 & 233 & & 235 & & \\
\hline \multicolumn{7}{|l|}{$\mathrm{CF}$} \\
\hline Q1 Changes in functioning & $88(6.6)$ & $82(35.2)$ & $7.7(5.5-10.9)$ & 185 (78.7) & $52.7(36.1-77.1)$ & $<0.001$ \\
\hline $\mathrm{Q} 2>1 \mathrm{~h}$ day sleeping & $82(6.1)$ & $50(21.5)$ & $4.2(2.9-6.2)$ & $86(36.6)$ & $8.9(6.3-12.6)$ & $<0.001$ \\
\hline Q3 > 1 h drowsy & $305(22.7)$ & $141(60.5)$ & $5.2(3.9-7.0)$ & $159(67.7)$ & $7.1(5.3-9.6)$ & 0.05 \\
\hline Q4 Difficult to arouse & 254 (18.9) & $106(45.5)$ & $3.6(2.7-4.8)$ & $135(57.5)$ & $5.8(4.3-7.8)$ & $<0.001$ \\
\hline \multicolumn{7}{|l|}{ RBD } \\
\hline Q5 Dream enact & $56(4.2)$ & $15(6.4)$ & $1.6(0.9-2.8)$ & $106(45.1)$ & $18.9(13.0-27.4)$ & $<0.001$ \\
\hline Q6 Dream enact* & $47(3.5)$ & $12(5.2)$ & $1.5(0.8-2.9)$ & $69(29.4)$ & $11.5(7.6-17.2)$ & $<0.001$ \\
\hline \multicolumn{7}{|l|}{ VH } \\
\hline Q7 Eye tricks* & $27(2.0)$ & $7(3.0)$ & $1.5(0.7-3.5)$ & $72(30.6)$ & $21.5(13.4-34.5)$ & $<0.001$ \\
\hline Q8 Sees things* & $32(2.4)$ & $16(6.9)$ & $3.0(1.6-5.6)$ & $98(41.7)$ & $29.3(18.9-45.3)$ & $<0.001$ \\
\hline Q9 False visions & $38(2.8)$ & $33(14.2)$ & $5.7(3.5-9.2)$ & $130(55.3)$ & $42.79(28.13-64.18)$ & $<0.001$ \\
\hline Q10 Sees things & $50(3.7)$ & $32(13.7)$ & $4.1(2.6-6.6)$ & $136(57.9)$ & $35.50(24.20-52.07)$ & $<0.001$ \\
\hline \multicolumn{7}{|l|}{ Motor symptoms } \\
\hline UPDRS 5-item score & $0.8 \pm 1.7$ & $2.9 \pm 2.7$ & $3.4(1.7-7.0)$ & $4.82 \pm 3.2$ & $19.68(11.64-33.28)$ & $<0.001$ \\
\hline
\end{tabular}

Unless marked by the *, which distinguishes questions posed to the patient, the questions are posed to the patient's caregiver. Data are reported as number of patients, $n(\%)$. UPDRS 5-item score data are reported as mean \pm standard deviation

Abbreviations: $C F$, cognitive fluctuations; $C I$, confidence interval; $O R$, odds ratio; $R B D$, REM sleep behavior disorder; $V H$, visual hallucinations; $Q$, questions. Data are reported as number of patients, $n(\%)$. The $p$-values* refer to test of comparison of the OR

Table 4 Stratification of patients according to LBCRS and AT-DLB toolkits

\begin{tabular}{llllcl}
\hline & $\begin{array}{l}\text { AT-DLB } \\
\text { No DLB }\end{array}$ & $\begin{array}{l}\text { AT-DLB } \\
\text { Possible DLB }\end{array}$ & $\begin{array}{l}\text { AT-DLB } \\
\text { Probable DLB }\end{array}$ & Total & \\
\hline LBCRS negative & 921 & 265 & 69 & 1255 & Frequency \\
& 50.88 & 14.64 & 3.81 & 69.34 & \% (whole cohort) \\
& $\mathbf{7 3 . 3 9}$ & 21.12 & 5.50 & & $\%$ (of the row) \\
& 88.30 & 59.55 & 21.43 & 555 & Frequency \\
LBCRS positive & 122 & 180 & 253 & 30.66 & $\%$ (whole cohort) \\
& 6.74 & 9.94 & 13.98 & & $\%$ (of the row) \\
& 21.98 & $\mathbf{3 2 . 4 3}$ & $\mathbf{4 5 . 5 9}$ & & (of the column) \\
Total & 11.70 & 40.45 & 78.57 & 1810 & \\
& 1043 & 445 & 322 & 100.00 & \\
\hline
\end{tabular}

Bold text: percentage of patients, whose diagnosis with AT-DLB and LBCRS was concordant

Abbreviations: $D L B$, dementia with Lewy bodies; $A T-D L B$, Assessment Toolkit for DLB; $L B C R S$, Lewy Body Composite Risk Score; LBCRS positive, probable DLB according to LBCRS; LBCRS negative, subjects who did not meet the criteria for probable DLB according to the LBCRS toolkit

\section{Comparison between results of the two questionnaires with the most recent DLB criteria (McKeith 2017)}

Results of each questionnaire, LBCRS and AT-DLB, were compared with the final diagnosis made by each Center, based only on the most recent criteria. In every group considered (non-DLB, possible DLB, and probable DLB), the percentage of the agreement between the two assessments and the final diagnosis based on international diagnostic criteria was higher than $93 \%$.
LBCRS and AT-DLB toolkits showed similar values of accuracy, sensitivity, and specificity, even though ATDLB seemed to be less efficient (Table 6). On the contrary, when simultaneously considered in the logistic models to assess the AUC, AT-DLB was significantly more informative (Supplementary Materials) in assessing possible $(0.13 \pm 0.02 p<0.001)$ and probable DLB $(0.10 \pm 0.01$ $p<0.001)$.

REM sleep behavior disorder was not correlated with possible DLB diagnosis, while it was directly correlated with probable DLB diagnosis. 
Table 5 Frequency of distribution for the items of AT-DLB, according to AT-DLB and LBCRS toolkits

\begin{tabular}{|c|c|c|c|c|c|c|}
\hline & $\begin{array}{l}\text { LBCRS negative } \\
\text { AT-DLB positive }\end{array}$ & $\begin{array}{l}\text { LBCRS positive } \\
\text { AT-DLB positive }\end{array}$ & $\begin{array}{l}\text { LBCRS negative } \\
\text { AT-DLB negative }\end{array}$ & $\begin{array}{l}\text { LBCRS positive } \\
\text { AT-DLB negative }\end{array}$ & $\begin{array}{l}\text { LBCRS positive } \\
\text { AT-DLB positive vs } \\
\text { LBCRS positive } \\
\text { AT-DLB negative }\end{array}$ & $\begin{array}{l}\text { LBCRS negative } \\
\text { AT-DLB negative } \\
\text { vs } \\
\text { LBCRS negative } \\
\text { AT-DLB positive }\end{array}$ \\
\hline Patients $(n)$ & 334 & 433 & 921 & 122 & & \\
\hline \multicolumn{7}{|l|}{$\mathrm{CF}$} \\
\hline $\begin{array}{l}\text { Q1 Changes in } \\
\text { functioning }\end{array}$ & $86(25.75)$ & $214(49.42)$ & $19(2.06)$ & $36(29.51)$ & $2.33(1.52-3.60)$ & $0.06(0.04-0.10)$ \\
\hline $\begin{array}{l}\text { Q2 > } 1 \text { h day sleep- } \\
\text { ing }\end{array}$ & 47 (14.07) & $135(31.18)$ & $14(1.52)$ & $22(18.03)$ & $2.06(1.25-3.41)$ & $0.09(0.05-0.17)$ \\
\hline Q3>1 h drowsy & $242(72.46)$ & $315(72.75)$ & $20(2.17)$ & $28(22.95)$ & $8.96(5.60-14.37)$ & $0.01(0.01-0.02)$ \\
\hline $\begin{array}{l}\text { Q4 Difficult to } \\
\text { arouse }\end{array}$ & $198(59.28)$ & $255(58.89)$ & $16(1.74)$ & $26(21.31)$ & $5.29(3.30-8.49)$ & $0.01(0.01-0.02)$ \\
\hline \multicolumn{7}{|l|}{ RBD } \\
\hline Q5 Dream enact & $24(7.19)$ & $115(26.56)$ & $12(1.30)$ & $26(21.31)$ & $1.34(0.82-2.16)$ & $0.17(0.08-0.35)$ \\
\hline Q6 Dream enact* & $20(5.99)$ & $68(15.70)$ & $15(1.63)$ & 25 (20.49) & $0.72(0.43-1.20)$ & $0.26(0.13-0.51)$ \\
\hline \multicolumn{7}{|l|}{$\mathrm{VH}$} \\
\hline Q7 Eye tricks* & $10(2.99)$ & 77 (17.78) & $1(0.11)$ & $18(14.75)$ & $1.25(0.72-2.18)$ & $0.04(0.01-0.28)$ \\
\hline Q8 Sees things* & $25(7.49)$ & $101(23.33)$ & $4(0.43)$ & $16(13.11)$ & $2.02(1.14-3.57)$ & $0.05(0.02-0.16)$ \\
\hline Q9 False visions & $26(7.78)$ & $150(34.64)$ & $5(0.54)$ & $20(16.39)$ & $2.70(1.61-4.54)$ & $0.07(0.03-0.17)$ \\
\hline Q10 Sees things & $28(8.38)$ & $157(36.26)$ & $7(0.76)$ & $26(21.31)$ & $2.10(1.31-3.38)$ & $0.08(0.04-0.19)$ \\
\hline \multicolumn{7}{|l|}{ Motor symptoms } \\
\hline $\begin{array}{l}\text { UPDRS 5-item } \\
\text { score }\end{array}$ & $1.59 \pm 1.59$ & $3.67 \pm 3.27$ & $0.34 \pm 0.90$ & $3.98 \pm 3.24$ & $0.97(0.92-1.03)$ & $0.45(0.40-0.51)$ \\
\hline
\end{tabular}

Unless marked by the *, which distinguishes questions posed to the patient, the questions are posed to the patient's caregiver

Abbreviations: $C F$, cognitive fluctuations; $R B D$, REM sleep behavior disorder; $V H$, visual hallucinations; $Q$, questions

Table 6 Analysis of sensitivity, specificity, accuracy, and AUC of the two toolkits vs. consensus criteria

\begin{tabular}{lllll}
\hline & $\begin{array}{l}\text { LBCRS vs. pos- } \\
\text { sible DLB }\end{array}$ & $\begin{array}{l}\text { LBCRS vs. prob- } \\
\text { able DLB }\end{array}$ & $\begin{array}{l}\text { AT-DLB possible vs. } \\
\text { possible DLB }\end{array}$ & $\begin{array}{l}\text { AT-DLB probable } \\
\text { vs. probable DLB }\end{array}$ \\
\hline Accuracy & 0.78 & 0.83 & 0.72 & 0.76 \\
Sensitivity & 0.53 & 0.85 & 0.13 & 0.75 \\
Specificity & 0.83 & 0.83 & 0.72 & 0.75 \\
AUC & $0.68 \pm 0.02$ & $0.84 \pm 0.01$ & $0.81 \pm 0.01^{*}$ & $0.94 \pm 0.008^{\wedge}$ \\
\hline
\end{tabular}

*Possible DLB: Comparison between LBCRS positive and AT-DLB possible

$\wedge$ Probable DLB: Comparison between LBCRS positive and AT-DLB probable

Abbreviations: $A U C$, area under curve

\section{Discussion}

Our results showed a prevalence of about $26 \%$ of possible or probable DLB diagnosis in the Dementia Centers as compared to the total diagnoses. With this second survey, the percentage of DLB diagnosis was comparable with the results of our first survey [4], higher than the prevalence reported in autopsy proven cohorts [1,4]. Our data indicate a higher frequency of in vivo DLB diagnosis also compared to other European countries, according to recent observations $[17,18]$. In a large UK's multicenter study,
DLB prevalence was $2.4-5.9 \%$ [17]. Despite a globally lower prevalence (3.4\%), compared to our observation, a recent Belgian study found significant differences, in terms of DLB prevalence, according to the patients' ethnicity [18]. DLB diagnosis was more frequent in NorthAfricans and Latin-American first-generation immigrants, compared to subjects born in Belgium [18]. Overall, these findings suggest a complex interplay between genetic and acquired factors that could underlie different epidemiological results for DLB. Furthermore, the high percentage of DLB diagnosis observed among the Italian Dementia Centers could be at least partially explained by the 
inclusion of cases of mixed dementia, which may have been classified as possible DLB [12,13], as clinical diagnostic criteria for this entity are indeed very unspecific [1].

We found a good agreement between the two questionnaires, especially for probable DLB diagnosis. Even though the two questionnaires were validated before the most recent criteria were published [1], both toolkits have reached a high concordance with the current international diagnostic criteria. LBCRS toolkit showed a better internal consistency, as compared to AT-DLB, whereas the latter showed a better performance in identifying individuals with probable DLB (Table 6).

As regards as AT-DLB toolkit, CF was the most relevant factor, among all variables, for the accuracy of the diagnosis. $\mathrm{CF}$ are not an easy feature to be assessed in clinical practice. Clinician Assessment of Fluctuation (CAF) [19] is a helpful tool for the clinicians to identify properly this typical symptom of DLB. The AT-DLB toolkit could represent a suitable alternative for $\mathrm{CF}$ assessment. Indeed, positive answer to the CF item of AT-DLB correlated with a higher risk of DLB diagnosis, only followed by VH items. As regards to LBCRS toolkit, and in accordance with literature data, which reports that $\mathrm{VH}$ are the most specific symptom in differentiating DLB from AD [20], VH were the most relevant symptom, followed by RBD, for the accuracy of diagnosis.

From a speculative point of view, the combination of the two toolkits could lead to a superior diagnostic accuracy for the evaluation of $\mathrm{CF}, \mathrm{VH}$, and $\mathrm{RBD}$, which are currently considered as core clinical features.

Limitations of this study are related to missing information during data collection, such as comorbidities and the final clinical diagnosis made for each patient recruited in each Center. This issue did not allow to estimate the presence of other dementias which could have been diagnosed as DLB (especially possible DLB). A further limitation is the low number of patients whose diagnose was corroborated through the study of biomarkers. Only a very low percentage of patients were studied by indicative or supportive biomarkers including DaT-SPECT, myocardial scintigraphy, FDGPET, quantitative EEG, polysomnography.

To conclude, standardization in the clinical assessment of DLB symptoms should be regarded as a priority, until the discovery of novel and optimal biomarkers.

Supplementary Information The online version contains supplementary material available at https://doi.org/10.1007/s10072-022-05987-z.

Acknowledgements Italian Dementia with Lewy bodies Study Group (DLB-SINdem):

Baschi Roberta $^{1}$, Fragiacomo Federica ${ }^{2}$, Galantucci Sebastiano ${ }^{3}$, Gaspari Caterina ${ }^{4}$, Gazzola Gianmarco ${ }^{2}$, Magnani Giuseppe ${ }^{3}$, Mazzon Giulia ${ }^{5}$, Mozzetta Stefano ${ }^{2}$, Ravanelli Carmela ${ }^{4}$, Ruggiero Marco ${ }^{4}$, Salute Pierpaolo ${ }^{4}$, Scamarcia Pietro Giuseppe ${ }^{3}$, Turla Marinella ${ }^{6}$, Verde Federico $^{7}$, Volontè Maria Antonietta ${ }^{3}$
${ }^{1}$ Section of Neurology, Department of Biomedicine, Neuroscience and Advanced Diagnostics, University of Palermo, Palermo, Italy

${ }^{2}$ Department of Neurosciences, University of Padova, Padova, Italy

${ }^{3}$ Unit of Neurology, IRCCS San Raffaele Scientific Institute, Milan, Italy

${ }^{4}$ Department of Neuroscience, Imaging and Clinical Sciences, University G. d'Annunzio of Chieti-Pescara, Chieti, Italy

${ }^{5}$ Neurology Unit, Department of Medicine, Surgery and Health Sciences, University Hospital and Health Services of Trieste, Trieste, Italy

${ }^{6}$ UO Neurologia ASST Valcamonica, Brescia, Italy

${ }^{7}$ Department of Neurology and Laboratory of Neuroscience, IRCCS Istituto Auxologico Italiano and Università degli Studi di Milano, Milano, Italy

Funding Open access funding provided by D'Annunzio University of Chieti - Pescara within the CRUI-CARE Agreement. This study was funded by the Italian Ministry of Health Research Grant Ricerca Finalizzata 2018 (RF-2018-12366209: Dementia with Lewy Bodies: toward a standardization of the diagnostic tools among the Italian Dementia Centers).

\section{Declarations}

\section{Ethical approval None.}

Informed consent Informed consent was obtained from each patient at each participating center.

Conflict of interest The authors declare no competing interests.

Open Access This article is licensed under a Creative Commons Attribution 4.0 International License, which permits use, sharing, adaptation, distribution and reproduction in any medium or format, as long as you give appropriate credit to the original author(s) and the source, provide a link to the Creative Commons licence, and indicate if changes were made. The images or other third party material in this article are included in the article's Creative Commons licence, unless indicated otherwise in a credit line to the material. If material is not included in the article's Creative Commons licence and your intended use is not permitted by statutory regulation or exceeds the permitted use, you will need to obtain permission directly from the copyright holder. To view a copy of this licence, visit http://creativecommons.org/licenses/by/4.0/.

\section{References}

1. McKeith IG, Boeve BF, Dickson DW, Halliday G, Taylor J-P, Weintraub D, Aarsland D, Galvin J, Attems J, Ballard CG et al (2017) Diagnosis and management of dementia with Lewy bodies: fourth consensus report of the DLB consortium. Neurology 89:88-100. https://doi.org/10.1212/WNL.0000000000004058

2. Thomas AJ, Mahin-Babaei F, Saidi M, Lett D, Taylor JP, Walker L, Attems J (2018) Improving the identification of dementia with Lewy bodies in the context of an Alzheimer's-type dementia. Alzheimers Res Ther 10:27. https://doi.org/10.1186/ s13195-018-0356-0

3. McKeith IG, Ferman TJ, Thomas AJ, Blanc F, Boeve BF, Fujishiro H, Kantarci K, Muscio C, O'Brien JT, Postuma RB et al (2020) Research criteria for the diagnosis of prodromal dementia with Lewy bodies. Neurology 94:743-755. https://doi.org/10.1212/ WNL.0000000000009323 
4. Bonanni L, Cagnin A, Agosta F, Babiloni C, Borroni B, Bozzali M, Bruni AC, Filippi M, Galimberti D, Monastero R et al (2017) The Italian dementia with Lewy bodies study group (DLB-SINdem): toward a standardization of clinical procedures and multicenter cohort studies design. Neurol Sci Off J Ital Neurol Soc Ital Soc Clin Neurophysiol 38:83-91. https://doi.org/10.1007/ s10072-016-2713-8

5. Galvin JE, Duda JE, Kaufer DI, Lippa CF, Taylor A, Zarit SH (2010) Lewy body dementia: the caregiver experience of clinical care. Parkinsonism Relat Disord 16:388-392. https://doi.org/10. 1016/j.parkreldis.2010.03.007

6. Prince M, Knapp M, Albanese E, Banerjee S, Dhanasiri S, Fernández J-L, Ferri C, McCrone P, Snell T, Stewart R (2007) Dementia UK: a report into the prevalence and cost of dementia. Alzheimer's Sochttps://www.alzheimers.org.uk/sites/default/files/ 2018-10/Dementia_UK_Full_Report_2007.pdf?fileID=2

7. Galvin JE (2015) Improving the clinical detection of Lewy body dementia with the Lewy Body Composite Risk Score. Alzheimer's Dement 1:316-324. https://doi.org/10.1016/j.dadm.2015.05.004

8. Thomas AJ, Taylor JP, McKeith I, Bamford C, Burn D, Allan L, O'Brien J (2018) Revision of assessment toolkits for improving the diagnosis of Lewy body dementia: the DIAMOND Lewy study. Int J Geriatr Psychiatry 33:1293-1304. https://doi.org/10.1002/gps.4948

9. Folstein MF, Folstein SE, McHugh PR (1975) Mini-mental state. J Psychiatr Res 12:189-198. https://doi.org/10.1016/0022-3956(75)90026-6

10. Walker MP, Ayre GA, Cummings JL, Wesnes K, McKeith IG, O'Brien JT, Ballard CG (2000) The Clinician Assessment of Fluctuation and the One Day Fluctuation Assessment Scale. Br J Psychiatry 177:252-256. https://doi.org/10.1192/bjp.177.3.252

11. Cummings JL, Mega M, Gray K, Rosenberg-Thompson S, Carusi DA, Gornbein J (1994) The Neuropsychiatric Inventory: comprehensive assessment of psychopathology in dementia. Neurology 44:2308-2314. https://doi.org/10.1212/wnl.44.12.2308

12. (2003) The Unified Parkinson's Disease Rating Scale (UPDRS): status and recommendations. Mov Disord 18:738-750. https://doi. org/10.1002/mds. 10473

13. Boeve BF, Molano JR, Ferman TJ, Lin S-C, Bieniek K, Tippmann-Peikert M, Boot B, St Louis EK, Knopman DS, Petersen RC et al (2013) Validation of the Mayo Sleep Questionnaire to screen for REM sleep behavior disorder in a community-based sample. J Clin Sleep Med JCSM Off Publ Am Acad Sleep Med 9:475-480. https://doi.org/10.5664/jcsm.2670

14. Hosmer D, Lemeshow S (1989) Applied logistic regression. In: Sons JW (ed) Statistics in Medicine. John Wiley \& Sons, Ltd, vol 10, pp 1162-1163

15. Tavakol M, Dennick R (2011) Making Sense of Cronbach's Alpha. International Journal of Medical Education 2:53-55. https://doi. org/10.5116/ijme.4dfb.8dfd

16. McKeith IG, Boeve BF, Dickson DW, Halliday G, Taylor J-P, Weintraub D, Aarsland D, Galvin J, Attems J, Ballard CG et al (2017) Diagnosis and management of dementia with Lewy bodies. Neurology 89:88-100. https://doi.org/10.1212/WNL.0000000000004058

17. Kane JPM, Surendranathan A, Bentley A, Barker SAH, Taylor J-P, Thomas AJ, Allan LM, McNally RJ, James PW, McKeith IG et al (2018) Clinical prevalence of Lewy body dementia. Alzheimers Res Ther 10:19. https://doi.org/10.1186/s13195-018-0350-6

18. Segers K, Benoit F, Meyts J-M, Glibert G, Levy S, Surquin M (2021) Dementia with Lewy bodies in first-generation immigrants in a European memory clinic. Acta Neurol Belg 121:219-223. https://doi.org/10.1007/s13760-020-01492-2

19. Van Dyk K, Towns S, Tatarina O, Yeung P, Dorrejo J, Zahodne LB, Stern Y (2016) Assessing fluctuating cognition in dementia diagnosis. Am J Alzheimers Dis Other Demen 31:137-143. https://doi.org/10.1177/1533317515603359

20. Tiraboschi P, Corso A, Guerra UP, Nobili F, Piccardo A, Calcagni ML, Volterrani D, Cecchin D, Tettamanti M, Antelmi L et al (2016) (123) I-2 $\beta$-carbomethoxy-3 $\beta$-(4-iodophenyl)-N(3-fluoropropyl) nortropane single photon emission computed tomography and (123) I-metaiodobenzylguanidine myocardial scintigraphy in differentiating dementia with lewy bodies from other dementias: a comparativ. Ann Neurol 80:368-378. https:// doi.org/10.1002/ana.24717

Publisher's note Springer Nature remains neutral with regard to jurisdictional claims in published maps and institutional affiliations.

\section{Authors and Affiliations}

\section{Mirella Russo ${ }^{1}$ - Claudia Carrarini ${ }^{1}$ - Angelo Di lorio ${ }^{2} \cdot$ Raffaello Pellegrino $^{2} \cdot$ Amalia Cecilia Bruni $^{3}$. Salvatore Caratozzolo ${ }^{4}$. Annalisa Chiari ${ }^{5}$. Stefano Pretta ${ }^{6}$. Camillo Marra ${ }^{7}$. Maria Sofia Cotelli ${ }^{8}$. Andrea Arighi ${ }^{9}$. Giorgio G. Fumagalli ${ }^{9}$. Tatiana Cataruzza ${ }^{10}$. Francesca Caso ${ }^{11}$. Cristina Paci ${ }^{12}$. Mara Rosso ${ }^{13}$. Serena Amici ${ }^{14}$. David Giannandrea ${ }^{15}$. Andrea Pilotto ${ }^{4}$. Simona Luzzi ${ }^{16}$. Annalisa Castellano ${ }^{17} \cdot$ Fabrizia D'antonio $^{18}$. Antonina Luca ${ }^{19}$. Giorgio Gelosa ${ }^{20}$. Tommaso Piccoli $^{21}$ - Marco Mauri ${ }^{22}$ - Federica Agosta ${ }^{23}$. Claudio Babiloni ${ }^{24,25}$. Barbara Borroni ${ }^{4} \cdot$ Marco Bozzali $^{26} \cdot$ Massimo Filippi $^{23} \cdot$ Daniela Galimberti ${ }^{9,27} \cdot$ Roberto Monastero $^{28}$. Cristina Muscio ${ }^{29}$. Lucilla Parnetti $^{30}$. Daniela Perani ${ }^{31}$. Laura Serra ${ }^{32}$. Vincenzo Silani ${ }^{33}$. Pietro Tiraboschi ${ }^{29}$. Annachiara Cagnin $^{34}$. Alessandro Padovani ${ }^{4}$. Laura Bonanni ${ }^{2}$. on Behalf of the Italian Dementia with Lewy bodies Study Group (DLB-SINdem)}

1 Department of Neuroscience, Imaging and Clinical Sciences, University G. d'Annunzio of Chieti-Pescara, Chieti, Italy

2 Department of Medicine and Aging Sciences, University G. d'Annunzio of Chieti-Pescara, Chieti, Italy

3 Regional Neurogenetic Centre, Department of Primary Care, ASP-CZ, Lamezia Terme, Italy

4 Neurology Unit, Department of Clinical and Experimental Sciences, University of Brescia, Brescia, Italy
5 U.O. Di Neurologia, Azienda Ospedaliera Universitaria Di Modena, Modena, Italy

6 Neuroimaging Research Unit, Division of Neuroscience, IRCCS San Raffaele Scientific Institute, Milan, Italy

7 Memory Clinic, Fondazione Policlinico Gemelli, IRCCS Università, Cattolica del Sacro Cuore, Rome, Italy

8 UO Neurologia ASST Valcamonica, Brescia, Italy 
9 UOSD Neurologia, Malattie Neurodegenerative, Fondazione IRCCS Ca' Granda Ospedale Maggiore Policlinico, Milano, Italy

10 Department of Medicine, Surgery and Health Sciences, Neurology Unit, University Hospital and Health Services of Trieste, Trieste, Italy

11 Unit of Neurology, IRCCS San Raffaele Scientific Institute, Milan, Italy

12 Division of Neurology, Ospedale Madonna del Soccorso, ASUR Marche, San Benedetto del Tronto-Ascoli, Piceno, Italy

13 Neurology Clinic, SS Annunziata Hospital of Savigliano, Savigliano, Italy

14 Cognitive Disorder and Dementia Unit, USL Umbria 1, Perugia, Italy

15 Neurologia E Stroke Unit, Ospedale Di Gubbio E Gualdo Tadino, Perugia, Italy

16 Department of Experimental and Clinical Medicine, Polytechnic University of Marche - Ospedali Riuniti, Ancona, Italy

17 CDCD IRCSS Neuromed Di Pozzilli, Isernia, Italy

18 Department of Human Neurosciences, Sapienza University of Rome, Rome, Italy

19 Department of Medical, Surgical Sciences and Advanced Technologies, GF Ingrassia, University of Catania, Catania, Italy

20 Cognitive Neuropsychology Center, Niguarda Hospital, Milan, Italy

21 Unit of Neurology, Department of Biomedicine, Neurosciences and Advanced Diagnostics, University of Palermo, Palermo, Italy
22 Department of Biotechnologies and Life Sciences, University of Insubria, Varese, Italy

23 Unit of Neurology, Neuroimaging Research Unit, Division of Neuroscience, IRCCS San Raffaele Scientific Institute and Vita-Salute San Raffaele University, Milan, Italy

24 Department of Physiology and Pharmacology "V. Erspamer, Sapienza University of Rome, Rome, Italy

25 Hospital San Raffaele Cassino (FR), Cassino, Italy

26 Department of Neuroscience, University of Turin, Turin, Italy

27 Department of Biomedical, Surgical and Dental Sciences, University of Milan, Milan, Italy

28 Section of Neurology, Department of Biomedicine, Neuroscience and Advanced Diagnostics, University of Palermo, Palermo, Italy

29 Neurology 5-Neuropathology Unit, Fondazione IRCCS Istituto Neurologico Carlo Besta, Milan, Italy

30 Laboratory of Clinical Neurochemistry, Section of Neurology, Department of Medicine and Surgery, University of Perugia, Perugia, Italy

31 In Vivo Human Molecular and Structural Neuroimaging Unit, Division of Neuroscience, IRCCS San Raffaele Scientific Institute, Milan, Italy

32 Neuroimaging Laboratory, Fondazione Santa Lucia, IRCCS, Rome, Italy

33 Department of Neurology and Laboratory of Neuroscience, IRCCS Istituto Auxologico Italiano and Università Degli Studi Di Milano, Milano, Italy

34 Department of Neurosciences, University of Padova, Padova, Italy 